\title{
Sexual Maturity Status at Neutering
}

National Cancer Institute

\section{Source}

National Cancer Institute. Sexual Maturity Status at Neutering. NCI Thesaurus. Code C158346.

A description of when the neutering event took place, relative to the subject's sexual maturation status. 